\title{
Surface characterization of feldspathic ceramic using ATR FT-IR and ellipsometry after various silanization protocols
}

Queiroz, José Renato Cavalcanti ; Benetti, Paula ; Ȯzcan, Mutlu ; de Oliveira, Luiz Fernando Cappa ; Della Bona, Alvaro ; Takahashi, Fernando Eidi ; Bottino, Marco Antonio

\begin{abstract}
OBJECTIVES: This study characterized the feldspathic ceramic surfaces after various silanization protocols. METHODS: Ceramic bars $(2 \mathrm{~mm} \times 4 \mathrm{~mm} \times 10 \mathrm{~mm})(\mathrm{N}=18)$ of feldpathic ceramic (VM7, VITA Zahnfabrik) were manufactured and finished. Before silane application, the specimens were ultrasonically cleaned in distilled water for $10 \mathrm{~min}$. The ceramic specimens were randomly divided into nine groups $(\mathrm{N}=2$ per group) and were treated with different silane protocols. MPS silane (ESPE-Sil, 3M ESPE) was applied to all specimens and left to react at $20^{\circ} \mathrm{C}$ for $2 \mathrm{~min}(\mathrm{G} 20)$. After drying, the specimens were subjected to heat treatment in an oven at $38^{\circ} \mathrm{C}$ (G38), $79^{\circ} \mathrm{C}(\mathrm{G} 79)$ or $100^{\circ} \mathrm{C}(\mathrm{G} 100)$ for $1 \mathrm{~min}$. Half of the specimens of each group were rinsed with water at $80^{\circ} \mathrm{C}$ for $15 \mathrm{~s}$ (G20B, G38B, G79B, G100B). The control group (GC) received no silane. Attenuated total reflection infrared Fourier transform analysis (ATR FT-IR) was performed using a spectrometer. Thickness of silane layer was measured using a spectroscopic ellipsometer working in the $\lambda=632.8 \mathrm{~nm}$ (He-Ne laser) at $70^{\circ}$ incidence angle. Surface roughness was evaluated using an optical profilometer. Specimens were further analyzed under the Scanning Electron Microscopy (SEM) to observe the topographic patterns. RESULTS: ATR FT-IR analysis showed changes in Si-O peaks with enlarged bands around $940 \mathrm{~cm}(-1)$. Ellipsometry measurements showed that all post-heat treatment actions reduced the silane film thickness (30.8-33.5 nm) compared to G20 (40 nm). The groups submitted to rinsing in hot water (B groups) showed thinner silane films (9.8-14.4 nm) than those of their corresponding groups (without washing) $(30.8-40 \mathrm{~nm})$. Profilometer analysis showed that heat treatments $(\mathrm{Ra} \approx$ $0.10-0.19 \mu \mathrm{m} ; \mathrm{Rq} \approx 0.15-0.26 \mu \mathrm{m})$ provided a smoother surface than the control group $(\mathrm{Ra} \approx 0.48 \mu \mathrm{m} ; \mathrm{Rq} \approx 0.65 \mu \mathrm{m})$. Similar patterns were also observed in SEM images. SIGNIFICANCE: Heat treatment after MPS silane application improved the silane layer network. Rinsing with boiling water eliminated the outmost unreacted regions of the silane yielding to thinner film thicknesses.
\end{abstract}

DOI: https://doi.org/10.1016/j.dental.2011.10.009

Posted at the Zurich Open Repository and Archive, University of Zurich

ZORA URL: https://doi.org/10.5167/uzh-75552

Journal Article

Accepted Version

Originally published at:

Queiroz, José Renato Cavalcanti; Benetti, Paula; Ȯzcan, Mutlu; de Oliveira, Luiz Fernando Cappa; Della Bona, Alvaro; Takahashi, Fernando Eidi; Bottino, Marco Antonio (2012). Surface characterization of feldspathic ceramic using ATR FT-IR and ellipsometry after various silanization protocols. Dental Materials, 28(2):189-196.

DOI: https://doi.org/10.1016/j.dental.2011.10.009 
Surface characterization of feldspathic ceramic using ATR FT-IR and ellipsometry after various silanization protocols

José Renato Cavalcanti de Queiroz ${ }^{\mathrm{a},{ }^{*}}$, Paulo Benetti ${ }^{\mathrm{a}}$, Mutlu Özcan ${ }^{\mathrm{b}}$, L.F.C. de Oliveira ${ }^{\mathrm{c}}$, Alvaro Della Bona ${ }^{\mathrm{d}}$, F.E. Takahashi ${ }^{\mathrm{a}}$, Marco Antonio Bottino ${ }^{\mathrm{a}}$

${ }^{a}$ São Paulo State University, Department of Prosthodontics and Dental Materials, São José dos Campos, Brazil

${ }^{b}$ University of Zurich, Dental Materials Unit, Center for Dental and Oral Medicine, Clinic for Fixed and Removable Prosthodontics and Dental Materials Science, Zurich, Switzerland ${ }^{c}$ Federal University of Juiz de For a, Department of Chemistry, Juiz de Fora, Brazil ${ }^{d}$ University of Passo Fundo, Department of Restorative Dentistry, Passo Fundo, Brazil

Short title: Surface characterization of feldspathic ceramic after silanization

${ }^{*}$ Corresponding author:

Dr. José Renato Cavalcanti de Queiroz

São Jose dos Campos Dental School

São Paulo State University (UNESP)

Praça Melvin Jones, 48, Jardim São Dimas

Zip Code: $12245-360$

São José dos Campos/SP

Brazil

Tel: +55-11-8841-8809; fax: +55-xxxx.

E-mail address: joserenatocq@hotmail.com (J. Queiroz) 


\section{ABSTRACT}

Objectives. This study characterized the feldspathic ceramic surfaces after various silanization protocols.

Methods. Ceramic bars $(2 \mathrm{~mm} \times 4 \mathrm{~mm} \times 10 \mathrm{~mm})(\mathrm{N}=18)$ of feldpathic ceramic (VM7, VITA Zahnfabrik) were manufactured and finished. Before silane application, the specimens were ultrasonically cleaned in distilled water for 10 min. The ceramic specimens were randomly divided into nine groups ( $n=2$ per group) and were treated with different silane protocols. MPS silane (ESPE-Sil, 3M ESPE) was applied to all specimens and left to react at $20^{\circ} \mathrm{C}$ for $1 \mathrm{~min}(\mathrm{G} 20)$. After drying, the specimens were subjected to heat treatment in an oven at $38^{\circ} \mathrm{C}(\mathrm{G} 38), 79^{\circ} \mathrm{C}(\mathrm{G} 79)$ or $100^{\circ} \mathrm{C}(\mathrm{G} 100)$ for $1 \mathrm{~min}$. Half of the specimens of each group were rinsed with water at $38^{\circ} \mathrm{C}, 79^{\circ} \mathrm{C}, 100^{\circ} \mathrm{C}$ for $15 \mathrm{~s}(\mathrm{G} 38 \mathrm{~B}, \mathrm{G} 79 \mathrm{~B}, \mathrm{G} 100 \mathrm{~B})$. The control group (GC) received no silane. Attenuated total reflection infrared Fourier transform analysis (ATR FT-IR) was performed using a spectrometer. Thickness of silane layer was measured using a spectroscopic ellipsometer working in the $\lambda=632.8 \mathrm{~nm}$ (He-Ne laser) at $70^{\circ}$ incidence angle. Surface roughness was evaluated using an optical profilometer. Specimens were further analyzed under the Scanning Electron Microscopy (SEM) to observe the topographic patterns.

Results. ATR FT-IR analysis showed changes in Si-O peaks with enlarged bands around $940 \mathrm{~cm}^{-1}$. Ellipsometry measurements showed that all post-heat treatment actions reduced the silane film thickness (30.8 - $33.5 \mathrm{~nm})$ compared to control group $(40 \mathrm{~nm})$. The groups submitted to rinsing in hot water (B groups) showed thinner silane films $(9.8-14.4 \mathrm{~nm})$ than those of their corresponding groups (without washing) $(30.8-33.5 \mathrm{~nm})$. Profilometer analysis showed that heat treatments $(\mathrm{Ra} \approx 0.10-0.19 \mu \mathrm{m} ; \mathrm{Rq} \approx$ 0.15-0-26 $\mu \mathrm{m}$ ) provided a smoother surface than the control group $(\mathrm{Ra} \approx 0.48 \mu \mathrm{m} ; \mathrm{Rq} \approx 0.65 \mu \mathrm{m})$. Similar patterns were also observed in SEM images.

Significance. Heat treatment after MPS silane application improved the silane layer network. Rinsing with boiling water eliminated the outmost unreacted regions of the silane yielding to thinner film thicknesses. Keywords: Adhesion; Ceramic; Silane coupling agent; Surface; Surface Characterization 


\section{Introduction}

The cementation process of a resin-bonded restoration is the final step of operative treatment after a series of procedures in dentistry. For adhesive cementation of glassy matrix ceramics, after etching with hydrofluoric acid gel (HF), it is a well-established method to use a silane coupling agent, a monomer with reactive organic radicals and hydro-soluble monovalent group. Silane coupling agents promote adhesion between inorganic phase of ceramic and organic phase of bonding agent applied on the ceramic surface through siloxane bonds. In addition, silane increases the surface energy of ceramic substrates and improves cement damping. These events consequently promote microscopic interactions between both parts. This approach made it possible today to adhere minimal invasive restorations to dental tissues that do not rely on mechanical retention principles.

The randomly distributed groups of silica oxides on the glass surface adsorb water as a hydroxyl group, forming a weak hydrogen bond [1]. It is also possible that other oxides present on ceramic surface absorb or adsorb water. In fact, there is a kind of competition between silane and water at available bond sites. Hydrogen bonds are formed during and after silanization but according to Plueddemann [2] there are also covalent bonds between silane and the substrate surface.

The poly-condensation of bifunctional silane monomers with the general formula of (R-O-) 3-Si-O-R, $\mathrm{R}$ being the first and $\mathrm{O}-\mathrm{R}$ the second silane functionality, can provide a highly cross-linked and reactive poly-organosiloxane layer to ceramics [1]. However, their hydrolytic degradation is still of concern [3-6]. Therefore, attempts are being made to increase their cross-linking [6-9]. Hydrolysis of silane in water diminishes the performance of silane and thereby, limits the lifetime of adhesive joints [3]. When small molecules of water penetrate into the adhesive interface, existing physical bonds are destroyed and from the untreated surfaces cracks may initiate yielding to detachment of the resin-based materials [3]. In

order to minimize this effect, prior to silane application, glass ceramic surfaces are etched with HF that selectively removes the glassy matrix. Consequently, with the increased surface roughness, micromechanical retention is facilitated for adhesive procedures. However, the high chemical toxicity, the 
possibility to produce insoluble silica-fluoride salts that can interfere with the resin bond and the fact that some high crystalline content ceramics may not benefit from HF etching, can be considered as some of the reasons to eliminate HF from the bonding procedure [6]. When the goal is to eliminate the HF etching procedure without jeopardizing the chemical reaction at the ceramic-silane-resin interfaces, the traditional bonding protocol for cementation or repairs needs to be modified to obtain the best silane effect in bonding resin to ceramic [10].

The silane drying conditions and post-heat treatment procedures reduces solvents and enhances the cross-linking reaction $[2,3,7,11,12]$. The extent of cross-linking is found to increase from the outer layers of the silane towards the glass surface with the corresponding increase in the mechanical and hydrothermal stability [2]. The thickness, orientation and cross-linking of the silane layers are determined by a number of factors, such as conditions of deposition, solution $\mathrm{pH}$, silane concentration, nature of solvents, hydrolyzing time, drying temperature, and time between silane application and bonding $[3,5,11]$.

Three different structural regions are proposed in the deposited silane film: a) a physiosorbed region; b) a chemisorbed region; and c) a chemically reacted region. The outermost physisorbed region, that is the bulk of the deposited silane, consists of weak oligomeric siloxanols that hydrolyzes easily and can be eliminated in room temperature at relative humidity. The chemisorbed region, consisting of stronger oligomeric siloxanols, offers a greater resistance to hydrolysis and can be eliminated at high temperatures in water. The most inner region that is close to the ceramic surface, is stable and resistant to extraction in hot water and may be regarded as the chemically reacted region of the silane film [13].

Heat treatment could be achieved either in an oven or by application of hot water. From clinical perspective, the latter may be more difficult to achieve in chairside applications. If the heat treatment in oven would result in similar silane quality, its application would be more convenient on ceramic surfaces prior to adhesive cementation procedures.

The objective of this study therefore, was to characterize the feldspathic ceramic surface after various silanization protocols including post-heat treatment in an oven or rinsing with hot water. The tested 
hypothesis was that heat treatment of the silane film would improve its chemical reactivity and the resistance to hydrolysis.

\section{Material and methods}

\subsection{Specimen preparation and experimental groups}

The brands, types, chemical compositions, manufacturers and batch numbers of the materials used in this study are listed in Table 1.

One dental technician made ceramic bars $(\mathrm{N}=18)$ of feldpathic ceramic (VM7, VITA Zahnfabrik, Bad Säckingen, Germany) according to the manufacturer's instructions. Initially, a standard acrylic resin with dimensions of $6 \mathrm{~mm} \times 8 \mathrm{~mm} \times 8 \mathrm{~mm}$ was made. From this acrylic, ceramic blocks were obtained measuring the same size for all groups. The pattern of acrylic resin was molded with a dense mass of the heavy part of polysiloxane (Elite HD, Zhermach, Badia Polesine, Italy). The powder (VITA VM7 Dentin 5M2, VITA Zahnfabrik) and modeling liquid (Modeling for Liquid Vita VM7, VITA Zahnfabrik) were mixed according to the manufacturer's instructions. The ceramic slurry was placed in small portions using a spatula, condensed inside the silicon impression and vibrated manually until completing the entire space of this impression. The excess fluid was removed with soft absorbent paper. Then, the ceramic block was removed from the tray. Finally, 18 bars of ceramic were obtained from a single impression. The blocks were sintered on the refractory base in the furnace (Vacumat 40, VITA Zahnfabrik) following the firing cycles recommended by the manufacturer. Considering that the contraction after sintering of ceramics is around $20 \%$, post-sintering blocks had approximate dimensions of $2 \mathrm{~mm} \times 4 \mathrm{~mm} \times 10 \mathrm{~mm}$.

Subsequently, ceramic specimens were ground finished to 1200 -grit silicon carbide metallographic abrasive papers (3M ESPE, St Paul, MN, USA) under water-cooling in a polishing machine (DP-10, Panambra, São Paulo, Brazil). Prior to the silane application, the specimens were ultrasonically cleaned (Vitasonic, Vita Zanhfabrik) in distilled water for 10 minutes and dried by oil-free air spray for 30 seconds. 
The ceramic specimens were randomly divided into nine groups ( $\mathrm{n}=2$ per group) and were treated with different silane protocols by one person. A 3-methacryloxypropyltrimethoxy silane (ESPE-Sil, 3M ESPE) was applied on the ceramic surfaces and left to react with the surface at a) $20^{\circ} \mathrm{C}$ in room temperature at $23 \%$ relative humidity (G20), b) $38^{\circ} \mathrm{C}(\mathrm{G} 38)$, c) $79^{\circ} \mathrm{C}(\mathrm{G} 79)$ d) $100^{\circ} \mathrm{C}(\mathrm{G} 100)$ for 1 minutes in an oven. Control group received no silane (GC). After drying, one of the specimens of each group (G20, G38, $\mathrm{G} 79, \mathrm{G} 100$ ) was rinsed with water at $20,38,79,100^{\circ} \mathrm{C}$ for 15 seconds (G20B, G38B, G79B, G100B).

\subsection{Silane film measurement}

The thickness of the silane film was determined using a Spectroscopic Ellipsometer (DRE-ELX02, Ratzeburg, German) working with $\lambda=632.8 \mathrm{~nm}$ (He-Ne laser) at $70^{\circ}$ incidence angle. The measurements were made after once after surface drying by oil-free air spray for 30 seconds and after rinsing with hot water. Since the ceramic specimens did not provide adequate light reflection, silane was applied onto smooth $(\mathrm{Ra}<10 \mathrm{~nm})$ and highly reflecting electropolished silicon specimens $(10 \mathrm{~mm} \times 10 \mathrm{~mm})$. The optical constants of the substrate were determined from measurements $(4 \mathrm{~s})$ of the ellipsometric parameters $(\psi$ and $\Delta)$ before silane application.

\subsection{Surface roughness analysis}

The average surface roughness $(\mathrm{Ra})$ and the root mean square roughness $(\mathrm{Rq})$ of the ceramic surfaces were measured before and after silane application. Roughness measurements were made in 3 different regions $(75 \mu \mathrm{m} \times 75 \mu \mathrm{m}$ ) of the ceramic surfaces in an optical profilometer (Wyko NT 1100, Veeco, Tucson, USA) by generating 3D images of the surfaces that were analyzed with the Wyko Vision 32 software (Veeco, USA). The vertical measurement range of the optical profilometer used was $0.1 \mathrm{~nm}$ to $1 \mathrm{~mm}$ with the vertical resolution of $\mathrm{Ra}<1 \AA$.

Specimens were further gold sputtered in a sputtering device and analyzed under the Scanning Electron Microscopy (SEM) (Zeiss DSM 950, Carl Zeiss, Jena, Germany) at $25 \mathrm{kV}$ to observe the topographic changes on silane treated surfaces.

\subsection{FT-IR analysis}


The Attenuated Total Reflection Infrared Fourier Transform (ATR FT-IR) analysis was performed using an FT-IR spectrophotometer (BOMEM MB-102, Quebec, Canada) and an optically dense zinc-selenide crystal.

The ceramic surface was placed in contact with the ATR crystal. An infrared beam was directed to a high refractive index crystal. The internal reflectance creates a passing wave that extends outside the crystal to the entire specimen surface that is in contact with the crystal. The attenuated energy from each passing wave returns to the IR beam that is located on the opposite side of the crystal. Once the IR beam is detected, the IR spectrometer system generates an infrared spectrum. All transmission spectra were recorded at $4 \mathrm{~cm}^{-1}$ resolutions in the range of $400-4000 \mathrm{~cm}^{-1}$. Sixty-four scans were made to obtain each spectrum.

\section{Results}

Ellipsometry measurements showed that all post-heat treatment actions reduced the silane film thickness (30.8 - $33.5 \mathrm{~nm}$ ) compared to control group (40 nm) (Table 2). The groups submitted to rinsing in hot water (B groups) showed thinner silane films $(9.8-14.4 \mathrm{~nm})$ than those of their corresponding groups (without washing) $(30.8-33.5 \mathrm{~nm})$.

The mean surface roughness values showed that all heat treatments, regardless of the temperature variations, provided a smoother surface than the control (GC) (Table 3).

The images from the optical profilometer (Figs. 1a-h) and SEM (Figs. 2a-h) illustrated variations in surface topographies. Small regions of silane accumulation were evident on the surfaces of specimens in G20 (Figs. 1a, 2a).

FT-IR results are demonstrated in Figs. 3a-d where absorbance is presented as the function of wave number $\left(\mathrm{cm}^{-1}\right)$. The infrared spectrum analysis showed differences between $\mathrm{G} 20$ and other groups. The band around $1080 \mathrm{~cm}^{-1}$ in G20 was due to asymmetric -Si-O-Si- (siloxane network in log chains) stretching vibrations, and $1164.9 \mathrm{~cm}^{-1}$ was due to $\mathrm{Si}-\mathrm{O}-\mathrm{CH}_{3}$. This has overlapped by a broad band with 
peaks in G38- $989.4 \mathrm{~cm}^{-1}$; G79- $985.5 \mathrm{~cm}^{-1}$; G100- $929.6 \mathrm{~cm}^{-1}$; G20B- $937.3 \mathrm{~cm}^{-1}$; G38B, G79B and 100B- $935.4 \mathrm{~cm}^{-1}$ due to strong Si-O bonds [14].

In all groups except G20, the O-H peak $\left(1637.4 \mathrm{~cm}^{-1}\right)$ disappeared due to complete evaporation of the solvent and hydrolysis of the sub-products (Fig. 3a). While $\mathrm{C}=\mathrm{O}$ (free carbonyl groups, $1718.4 \mathrm{~cm}^{-1}$ ) and $\mathrm{CH}_{2}$ peaks $\left(\mathrm{CH}\right.$ bending vibration, $\left.1296 \mathrm{~cm}^{-1}\right)$ that appeared in $\mathrm{G} 20$ (Fig. 3a) decreased, $\mathrm{CH}_{3}(\mathrm{CH}$ bending vibration, $1390 \mathrm{~cm}^{-1}$ ) increased in other FT-IR spectra (Figs. 3b-d), indicating organic polymerization reaction.

\section{Discussion}

This study was undertaken to characterize the feldspathic ceramic surface after various post-heat treatment protocols for silanization. Silane application is an important step when adhering organic materials to inorganic ones. However, silanization process is considered technique-sensitive and its instability may cause deterioration in adhesion $[9,12]$. The quality of adhesion using silane coupling agents does not only depend on the specifications of the silane such as solution $\mathrm{pH}$, concentration, the nature of the solvent, hydrolysis time but also on the protocol of application such as drying conditions, time between application of silane and adhesive resin, temperature and humidity of the environment $[5,15]$. Based on the results of this study, the tested hypothesis that heat treatment of the silane film would improve its chemical reactivity and the resistance to hydrolysis could be accepted.

It has been previously demonstrated that silane adhesion decreases as the relative humidity of the air increases [16]. Ambient conditions may affect the water desorption rate where the amount of water present in the environment influences the rate of several competing reactions during silane condensation reaction. Although this aspect has not been studied in depth, in this study, silane was applied at room temperature at $23 \%$ relative humidity and then the specimens were subjected to further heat treatment conditions either in the oven or by hot water application. 
In a previous study, the application of an MPS silane containing ethanol as a solvent using a brush did not induce any morphological changes on the glass surface before and after thermal treatments [17]. Qualitative observations in this study using SEM and optical profilometry also showed in general uniform, smooth silane coating, except for G20. In G20, it seemed that the silane was not uniformly distributed on the ceramic surface that may be as a consequence of silane excess, incomplete formation of poly-organosilanes or adsorption of the solvent. Failure in the poly-organosilane formation can facilitate hydrolysis and degradation. This was also observed when silanized interfaces were subjected to water storage or thermocycling $[18,19]$. In aqueous environment, water hydrolyzes the silane film, breaking the siloxane bonds of molecules, decreasing the bond to inorganic surfaces [1]. Since the specimens treated above $20^{\circ} \mathrm{C}$ did not show this kind of non-uniformity, the effect of heat treatment was evident. However, interestingly already at $38^{\circ} \mathrm{C}$ that was in fact representing the body temperature in this study, uniform surface texture was also reached. Silane in G20 presented the highest film thickness with $40 \mathrm{~nm}$ but subsequent rinsing with water decreased the film thickness (G20B) to $9.8 \mathrm{~nm}$.

Several theories have been proposed to explain the effect of heat treatment on the silane film: a) an enhanced inability of water to diffuse through the silane film due to increased cross-linking $[5,19]$, b) oven heating could ensure complete bond formation between the silica matrix and the silane, resulting in oligomerization [19,20], c) evaporate solvent and volatilize products formed during condensation of the silanol groups in dry condition, could create large internal pressure gradients [9,21,22], and d) heating at $100^{\circ} \mathrm{C}$ could consolidate 3 regions of silane film into a innermost region without any interphase region [22]. The last theory was not in accordance with the results obtained in this study (Why?).

The silane film thickness is of importance in bond strength of resin-based materials to ceramics. An excessive amount of coupling agent cannot be efficient to provide a strong bond since in thick films, interphase properties may be impaired [4]. On the other hand, it is also true that development of a thicker silane film with stronger network can be very useful. There is an optimum thickness of this region or interphase in the coupling agent, with maximum siloxane bond formation capable of resisting 
hydrolysis degradation and absorbing mechanical stress without crack growth into the silane film. The chemically reacted region, which is the innermost layer, is mainly responsible for resisting to hydrothermal attack. Since the outmost layer is not chemically bonded to the internal layer, it will be ineffective in promoting adhesion. Therefore, the outmost silane film layer can be removed by rinsing procedures. The ellipsometry results showed that after rinsing procedures, silane film thickness decreased compared to those of the non-rinsed groups. In addition, not only $79^{\circ} \mathrm{C}$ which is the alcohol or solvent or $100^{\circ} \mathrm{C}$ water which is sub-product evaporation temperature [7], at $20^{\circ} \mathrm{C}$ and $38^{\circ} \mathrm{C}$ rinsing also decreased film thickness was observed. Elimination of the outmost region was presented in earlier studies after rinsing water at $80^{\circ} \mathrm{C}[3,7,11]$. Low film thickness other than $80^{\circ} \mathrm{C}$ in this study could be partially due to the methodology employed. Initially, we have performed ellipsometry measurements on the ceramic surface. However, the roughness spread the surface laser and the data collected were not reproducable. Then, we used the silicon specimens with surface roughness of $<10 \mathrm{~nm}$. The use of silicon plates to determine the silane thickness could be founded in other studies $[x, x]$. Yet, the roughness and topography of such surfaces are surely different compared to ceramic surfaces which could be further compared using other methods.

Although Atomic Force Microscopy (AFM) is a spectacular technique, with this method surface analysis is achieved in the contact mode. Optical profilometer used in this study is a rapid method that allows for non-destructive, and non-contact surface analysis. The interference microscope or optical profilometer measures the surface roughness where the light intensity of the fringes is related to the surface height. It has a sub-nanometer vertical resolution with a dynamic range that can be improved depending on the scan size, greatly exceeding other probe microscopes. It provides fast images of the surface such as SEM with roughness parameter supported by 3D images similar to AFM. Nonetheless, the results could be verified with AFM to observe the effect of methodological differences.

Not only the thickness but also the network quality of silane film is important. FT-IR spectra can be used to provide useful information about the presence or absence of functional groups $[4,7,21,23,24]$. 
This study used ATR FT-IR technique that allows the direct (in situ) identification of silane film as well as the amount of each component. Spectra of G20 and G20B showed a band at $1637 \mathrm{~cm}^{-1}$, referring to $\delta(\mathrm{O}-\mathrm{H})$, which disappeared in other IR spectra. Solvent evaporation represents a factor affecting the coupling potential of silanes. Even though a small amount of solvent may help silane wetting, incomplete evaporation may impair adhesion. Water, alcohol, acetone, acetic acid and other by-products that remain in the pores of the network, if not completely expelled by drying procedure, may remain as hydrogen-bonded to the $\mathrm{OH}$-rich silica fillers of the composite substrate. This may decrease the number of bond sites available for reacting with silane, thus compromising the final degree of siloxane bond formation $[4,15,21]$. The spectra of all temperatures above $38^{\circ} \mathrm{C}$ suggest that the heat treatment helps in the evaporation of solvent and volatile sub-products from the silane reaction, catalyzing and completing the condensation reactions both with the substrate and within the silane coating.

The spectra of silane of G79, G79B and G100, G100B showed pattern of bands only with intensity variation but without wave number shifts. All the spectra strongly suggest that this procedure is efficient to remove the outmost region in silane film, which is in agreement with the ellipsometry analysis. In general, the intensity of several bands increased at higher temperatures, suggesting that increasing temperature is efficient to promote the cross-linking network in silane film [15].

The heat treatment seemed to provide the appearance of strong absorption bands in the spectra, as it can be seen for strong siloxane network bonding on the feldsphatic ceramic surface (-Si-O-Si $\left.i_{(\text {(eramic) }}\right)$ in the range around $1000 \mathrm{~cm}^{-1}$. The broad band centred at $940 \mathrm{~cm}^{-1}$ was probably overlapping with the other bands at $1164 \mathrm{~cm}^{-1}$ (methoxy group) and $1080 \mathrm{~cm}^{-1}$ (-Si-O-Si-O-Si-). This can be associated to an improvement of the network resistance to hydrolysis degradation by initial complete hydrolysis of methoxy group present in solution, besides the energy which is necessary to break the long chains of Si-O-Si- in lower $-\mathrm{Si}-\mathrm{O}-\mathrm{Si}_{(\text {(ceramic) }}$ chains.

In the spectra, the band at $1296 \mathrm{~cm}^{-1}$, assigned to the ester group, decreased after heat treatment. On the order hand, an increase in the intensity of the $1390 \mathrm{~cm}^{-1}$ band, assigned to $-\mathrm{C}-\mathrm{H}$ deformation, 
strongly indicated that silane organic radical could react each order, helping the stability of silane film. In fact, water and organic solvent can penetrate into the pores of the glassy material during drying phase. While some of the larger pores could not be filled, smaller pores can be filled with solvent. Since these small pores remain sealed, the presence of solvent can create great internal pressure gradients. This process could cause cracking and fracture in interphase [21].

Heat treatment and rinsing at $80^{\circ} \mathrm{C}$ water improved bond strength between organic to inorganic elements after water storage or thermocycling compared to those groups that did not receive any postheat treatment $[6,7,9,15,22,24,25]$. The curing temperature and the boiling point of the silanes may vary between silanes. According to the manufacturer's information, boiling point of ESPE-Sil was $78^{\circ} \mathrm{C}$ at 1 atm. Boiling point of methacrylate silanes may also vary between products. To answer this question, other qualitative analysis such as termogravimetry needs to be made. However, since $100^{\circ} \mathrm{C}$ exceeds the boiling temperature of the silane studied, post-heat treatment below this temperatures could be considered safer for this silane. This may however not count for other silanes. In this study, the silane layer diminished the surface roughness of the ceramics. Adhesion studies need to verify whether it has an impact on the bond strength of principally the bonding agent and subsequently the resin cement. Also marginal integrity and quality of adhesively restorations should be observed with the studied silanization protocols.

In this study only MPS silane was used that is the most frequently used silane type in dentistry. It is also more biocompatible compared to many other industrial silanes. This could be considered as a limitation of this study. In future studies, the post-heat treatment parameters need to be verified for other silanes. Also, the effect of silane post-treatment parameters with and without HF acid gel on glassymatrix ceramics on bond strength of resin materials warrants future research to be able to make a clinical recommendation for the optimum silanization protocol. 


\section{Conclusions}

From this study, the following could be concluded:

1. Post-heat treatment of the studied MPS silane at $38,79,100^{\circ} \mathrm{C}$ improved the quality of siloxane network on the feldspathic ceramic surface compared to drying at room temperature $\left(20^{\circ} \mathrm{C}\right)$, also yielding to thinner silane film thicknesses.

2. Rinsing the MPS silane treated surfaces with hot water for 15 seconds removed the unreacted region of the silane and the remained film formed an adhesive interphase with more stable chemical characteristics and resistance to the hydrolytic degradation. 


\section{References}

[1] Olmos D, González-Benito J, Aznar AJ, Baselga J. Hydrolytic damage study of the silane coupling region in coated silica microfibres: $\mathrm{pH}$ and coating type effects. J Mater Process Tech 2003;143144:82-6.

[2] Plueddemann EP. Silane coupling agents. New York, Plenum press; 1991.

[3] Ishida H, Koenig JL. Effect of hydrolysis and drying on the siloxane bonds of a silane coupling agent deposited on E-glass fibers. J Polym Sci: Part B Polym Phys 1980;18:233-7.

[4] Chua PS, Dai SR, Piggott MR. Mechanical properties of the glass fiber-polyester interphase. Part 2-effect of water on debonding. J Mater Sci 1992;27:919-24.

[5] Abel ML, Allington LD, Digby RP, Porritt N, Shaw SJ, Watts JF. Understanding the relationship between silane application conditions, bond durability and locus of failure. Int J Adhes Adhesives 2006;26:2-15.

[6] Fabianelli A, Pollington S, Papacchini F, Goracci C, Cantoro A, Ferrari M, van Noort R. The effect of different surface treatments on bond strength between leucite reinforced feldspathic ceramic and composite resin. J Dent 2010;38:39-43.

[7] Hooshmand $T$, van Noort R, Keshvad A. Bond durability of the resin-bonded and silane treated ceramic surface. Dent Mater 2002;18:179-88.

[8] Shen C, Oh W, Williams JL. Effect of post-silanization drying on the bond strength of composite to ceramic. J Prosthet Dent 2004;91:453-8.

[9] Monticelli F, Toledano M, Osorio R, Ferrari M. Effects of temperature o the silane coupling agents when bonding core resin to quartz fiber post. Dent Mater 2006;22:1024-8.

[10] Della Bona A. Adesão as cerâmicas: evidências científicas para uso clínico. São Paulo, Artes Médicas; 2009. 
[11] Ishida $H$, Koenig $J L$. An investigation of the coupling agent/matrix interface of fiberglass reinforced plastic by fourier transform infrared spectroscopy. J Polym Sci: Part B Polym Phys $1979 ; 17: 615-26$.

[12] Özcan M, Matinlinna JP, Vallittu PK, Huysmans MC. Effect of drying time of 3methacryloxyprophyl-trimethoxysilane on the shear bond strength of a composite resin to silicacoated base/noble alloy. Dent Mater 2004;20:586-90.

[13] Schrader ME. Radioisotopic studies of bonding at the interface. J Adhesion 1970;2:202-12.

[14] Silverstein. Spectroscopy chemistry NMR FTIR MS; Chapter 3, page 108, City, Country.

[15] Papacchini F, Monticelli F, Hasa I, Radovic I, Fabianelli A, Polimeni A, Ferrari M. Effect of airdrying temperature on the effectiveness of silane primers and coupling blends in the repair of a microhybrid resin composite. J Adhes Dent 2007;9:391-7.

[16] Underhill PR, Goring G, DuQuesnay DL. The effect of humidity on the curing of 3glycidoxypropyltrimetoxy silane. Int J Adhes Adhesives 2000;20:195-9.

[17] Pallavicini P, Dacarro G, Galli M, Patrini M. Spectroscopic evaluation of surface functionalization efficiency in the preparation of mercaptopropyltrimethoxysilane self-assembled monolayers on glass. J Colloid Interface Sci 2009;332:432-8.

[18] Roulet JF, Soderholm KJM, Longmate J. Effects of treatment and storage conditions on ceramic/composite bond strength. J Dent Res 1995;74:381-7.

[19] Matinlinna JP, Lassila LVJ, Özcan M, Yli-Urpo A, Vallittu PK. An introduction to silanes and theirs clinical applications in dentistry. Int J Prosthodont 2004;17:155-64.

[20] Bertelsen CM, Boerio FJ. Linking mechanical properties of silanes to their chemical structure: an analytical study of y-GPS solutions and films. Prog Organic Coat 2001;41:239-46.

[21] El-Nahhal IM, El-Ashgar NM. A review on polysiloxane-immobilized ligand systems: Synthesis, characterization and application. J Organometallic Chem 2007;692:2861-86. 
[22] Hooshmand T, van Noort R, Keshvad A. Storage effect of a pre-activated silane on the resin to ceramic bond. Dent Mater 2004;20:635-42.

[23] Frost RL, Mendelovici E. Modification of fibrous silicates surfaces with organic derivatives: an infrared spectroscopic study. J Colloid Interf Sci 2006;294:47-52.

[24] Sirisinha K, Chimdist S. Comparison of techiniques for determining crosslinking in silane-water crosslinked materials. Polym Test 2006;25:518-26.

[25] Barghi N, Berry T, Chung K. Effects of time and heat treatment of silanated porcelain on the bond strength. J Oral Rehabil 2000;27:407-12. 


\section{Captions to tables and figures:}

\section{Tables}

Table 1. The brands, types, chemical compositions, manufacturers and batch numbers of the materials used in this study.

Table 2. Silane film thickness $(\mathrm{nm})$ after different silanization protocols: at $20^{\circ} \mathrm{C}$ in room temperature (G20), $38^{\circ} \mathrm{C}(\mathrm{G} 38), 79^{\circ} \mathrm{C}(\mathrm{G} 79), 100^{\circ} \mathrm{C}(\mathrm{G} 100)$ for 1 minute in an oven. B groups represent specimens rinsed with water at $20,38,79,100^{\circ} \mathrm{C}$ for 15 seconds.

Table 3. Mean $\mathrm{Ra}$ and $\mathrm{Rq}$ values $(\mu \mathrm{m})$ after different silanization protocols. For group abbreviations see Table 2.

\section{Figures}

Figs. 1a-h 3D surface images of specimens obtained from optical profilometer in groups a) G20; b) G20B; c) G38; d) G38B; e) G79; f) G79B; g) G100; and h) G100B. Note the non-uniform silane film in G20 compared to those of other groups.

Figs. 2a-h SEM micrographs (500x) of silanized surfaces of a) G20; b) G20B; c) G38; d) G38B; e) G79; f) G79B; g) G100; and h) G100B. Note small regions of silane accumulation on the surface of the specimens in $\mathrm{G} 20$.

Figs. 3a-d ATR-FTIR spectra of MPS silane with wave numbers in $\mathrm{cm}^{-1}$ : a) G20, G20B; b) G38, G38B; c) G79, G79B; d) G100, G100B. 


\section{Tables}

Table 1. The brands, types, chemical compositions, manufacturers and batch numbers of the materials used in this study.

\begin{tabular}{|c|c|c|c|c|}
\hline Brand & Type & $\begin{array}{c}\text { Chemical Composition } \\
\text { Manufacturer }\end{array}$ & $\begin{array}{c}\text { Batch } \\
\text { Number }\end{array}$ \\
\hline VM7 & $\begin{array}{c}\text { Feldspathic } \\
\text { ceramic }\end{array}$ & $\begin{array}{c}\text { Glass frits melted in metal } \\
\text { oxides }\end{array}$ & $\begin{array}{c}\text { Vita } \\
\text { Zanhfabrik, } \\
\text { Bad } \\
\text { Säckingen, } \\
\text { Germany }\end{array}$ & 7404 \\
\hline ESPE-Sil & $\begin{array}{c}\text { Silane coupling } \\
\text { agent }\end{array}$ & $\begin{array}{c}\text { Ethyl alcohol, 3- } \\
\text { methacryloxypropyltrimethoxy, } \\
\text { methyl ethyl ketone }\end{array}$ & $\begin{array}{c}\text { 3M ESPE, St. } \\
\text { Paul, MN, } \\
\text { USA }\end{array}$ & 286318 \\
\hline
\end{tabular}

Table 2. Silane film thickness $(\mathrm{nm})$ after different silanization protocols: at $20^{\circ} \mathrm{C}$ in room temperature (G20), $38^{\circ} \mathrm{C}(\mathrm{G} 38), 79^{\circ} \mathrm{C}(\mathrm{G} 79), 100^{\circ} \mathrm{C}(\mathrm{G} 100)$ for 1 minute in an oven. B groups represent specimens rinsed with water at $20,38,79,100^{\circ} \mathrm{C}$ for 15 seconds.

\begin{tabular}{|c|c|c|c|c|c|c|c|c|}
\hline Silanization Protocol & G20 & G20B & G38 & G38B & G79 & G79B & G100 & G100B \\
\hline Film Thickness & 40 & 9.8 & 32.6 & 11 & 30.8 & 11.4 & 33.5 & 14.4 \\
\hline
\end{tabular}

Table 3. Mean $\mathrm{Ra}$ and $\mathrm{Rq}$ values $(\mu \mathrm{m})$ after different silanization protocols. For group abbreviations see Table 2.

\begin{tabular}{|c|c|c|c|c|c|c|c|c|c|}
\hline & Control & G20 & G20B & G38 & G38B & G79 & G79B & G100 & G100B \\
\hline $\mathrm{Ra}$ & 0.48 & 0.20 & 0.19 & 0.16 & 0.11 & 0.13 & 0.11 & 0.11 & 0.10 \\
\hline $\mathrm{Rq}$ & 0.65 & 0.27 & 0.26 & 0.24 & 0.15 & 0.17 & 0.16 & 0.16 & 0.15 \\
\hline
\end{tabular}




\section{Figures}

Figs. 1a-h 3D surface images of specimens obtained from optical profilometer in groups a) G20; b) G20B; c) G38; d) G38B; e) G79; f) G79B; g) G100; and h) G100B. Note the non-uniform silane film in G20 compared to those of other groups.

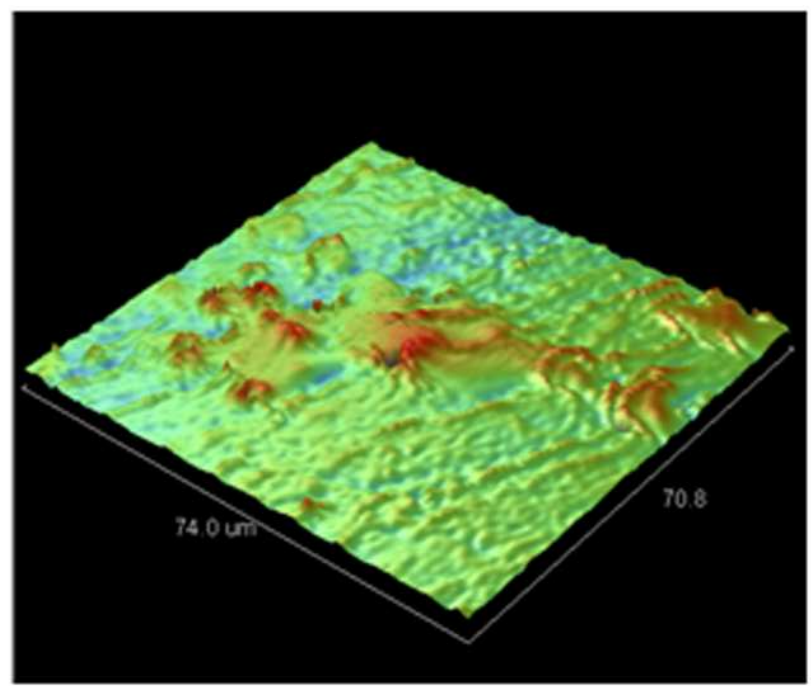

a)

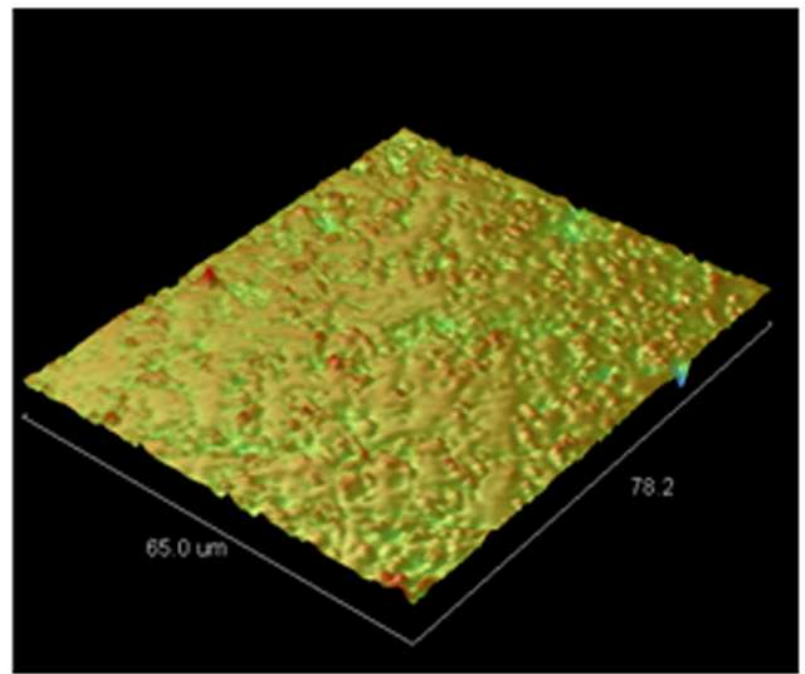

b) 

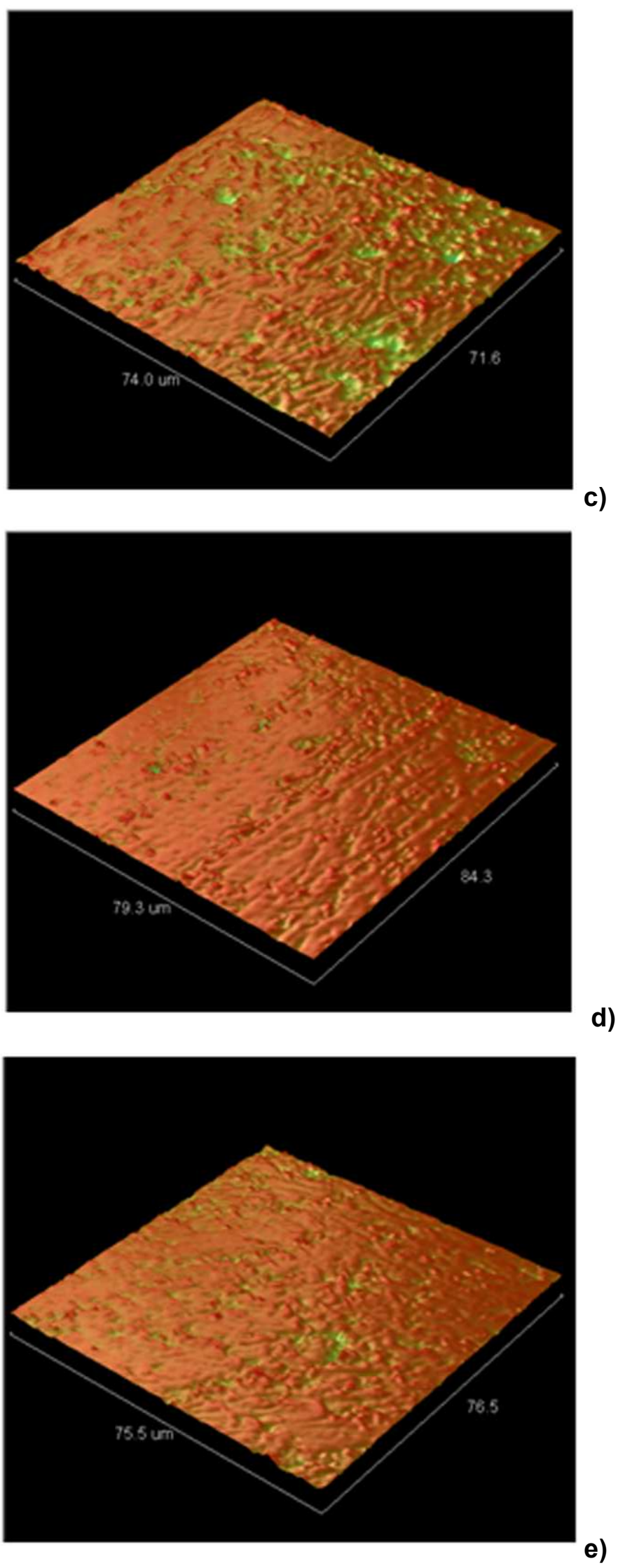

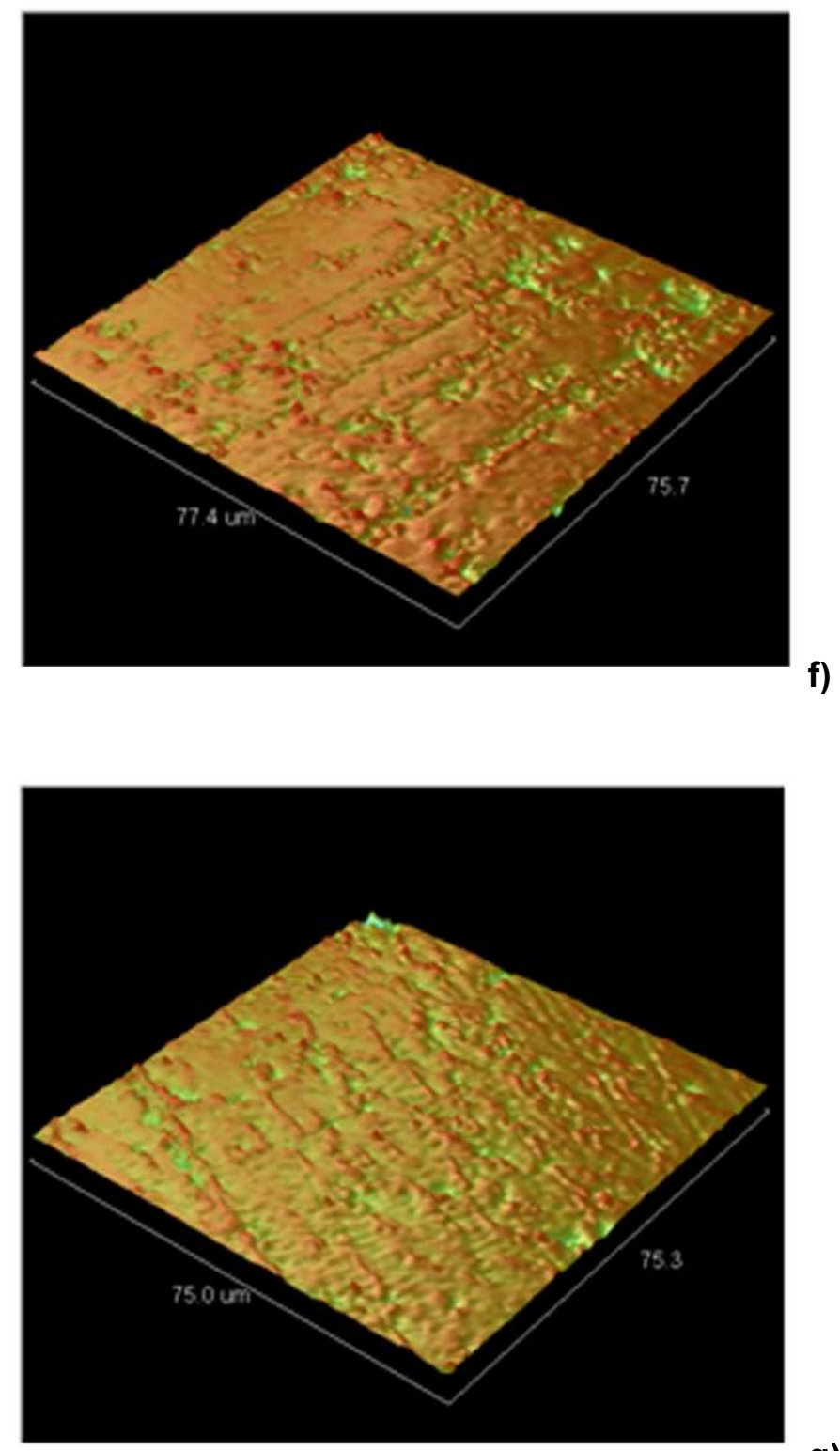

g)

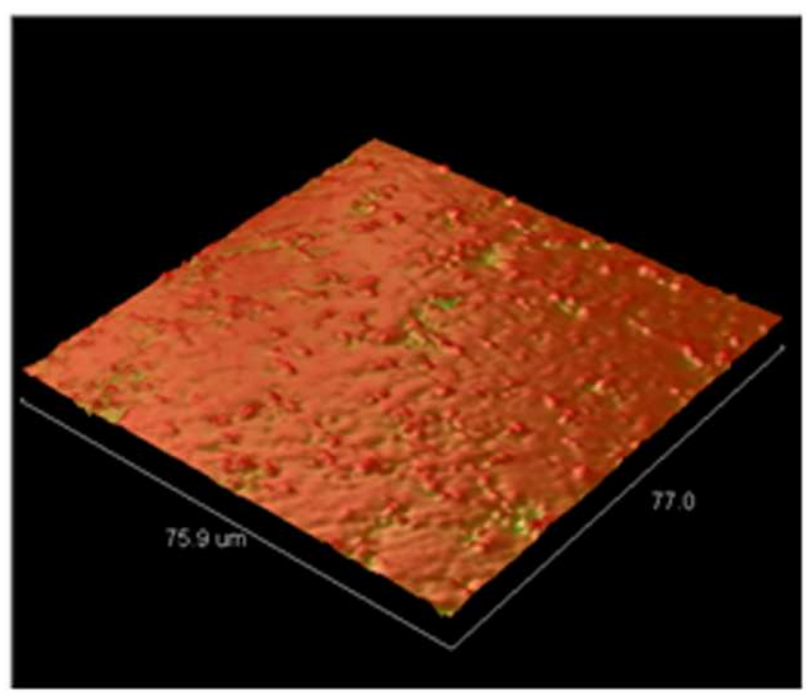

h) 
Figs. 2a-h SEM micrographs (500x) of silanized surfaces of a) G20; b) G20B; c) G38; d) G38B; e) G79; f) G79B; g) G100; and h) G100B. Note small regions of silane accumulation on the surface of the specimens in G20.
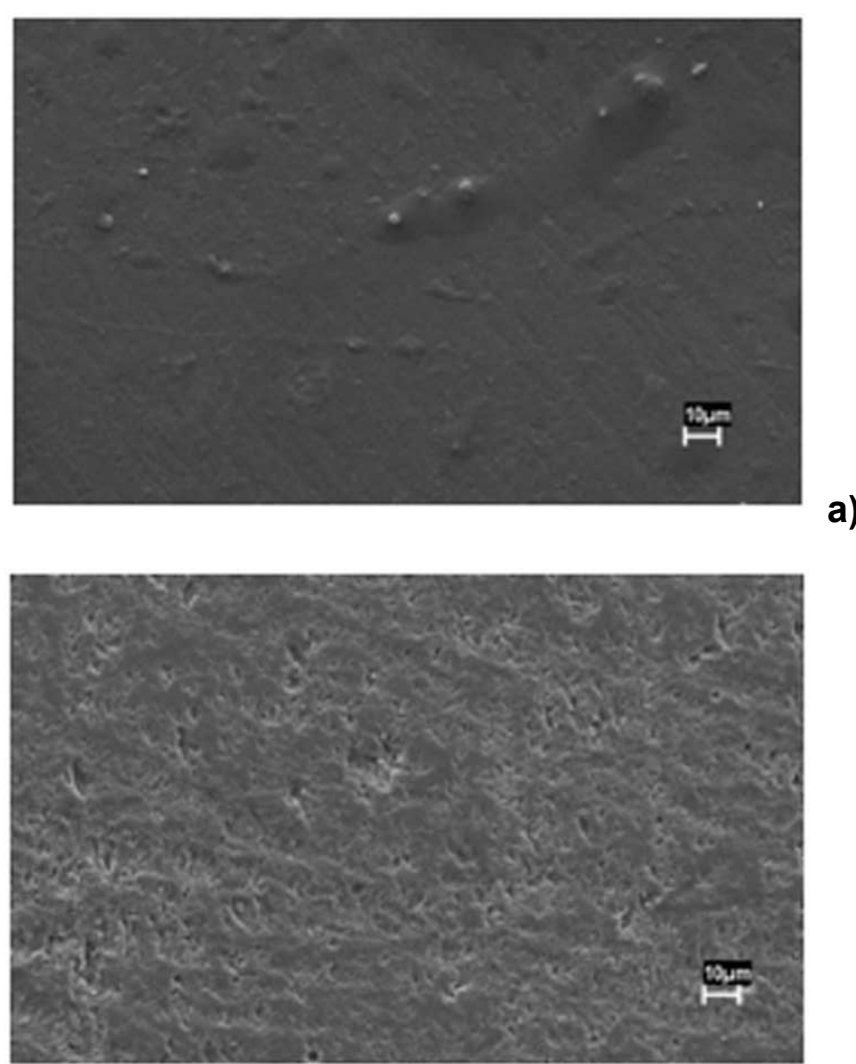

a)

b)

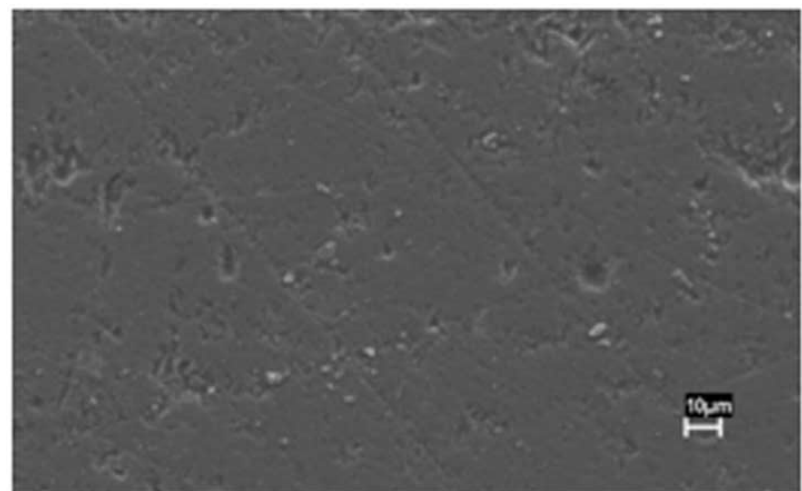

c)

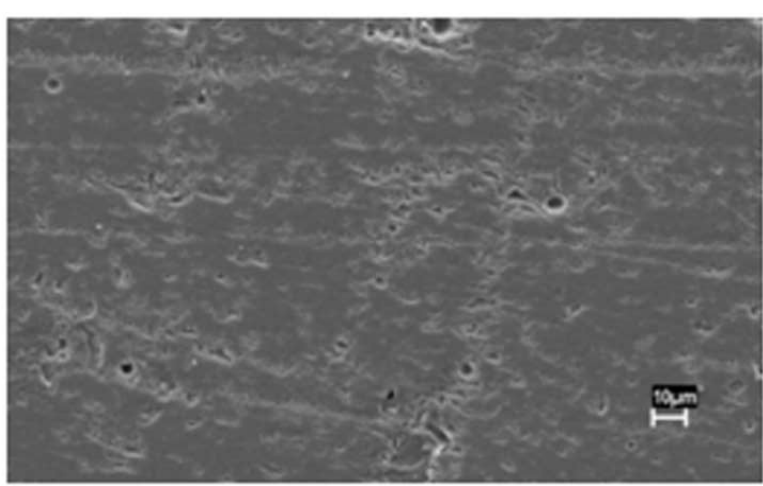

d) 

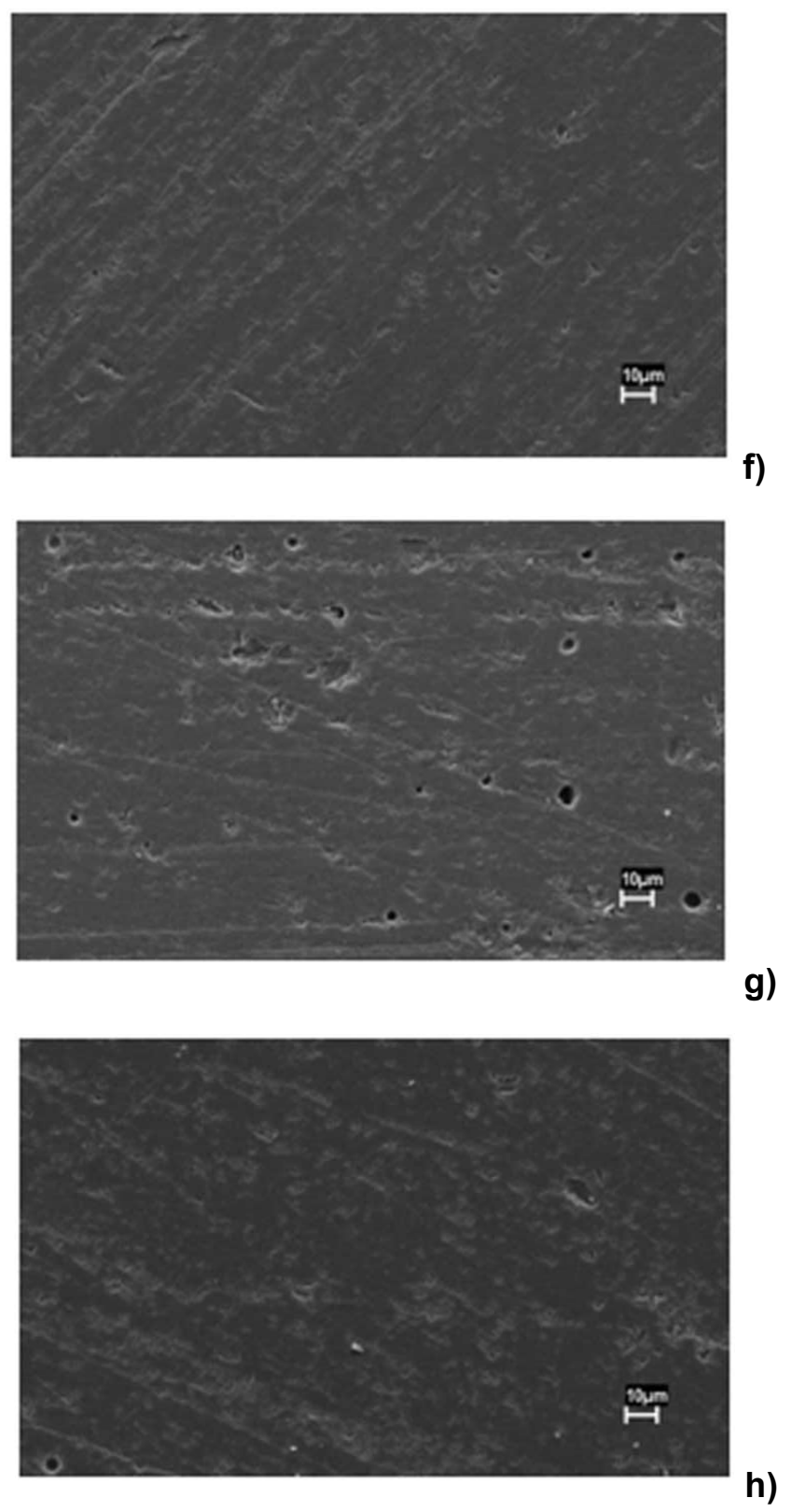
Figs. 3a-d ATR-FTIR spectra of MPS silane with wave numbers in $\mathrm{cm}^{-1}$ : a) G20, G20B; b) G38, G38B; c) G79, G79B; d) G100, G100B.

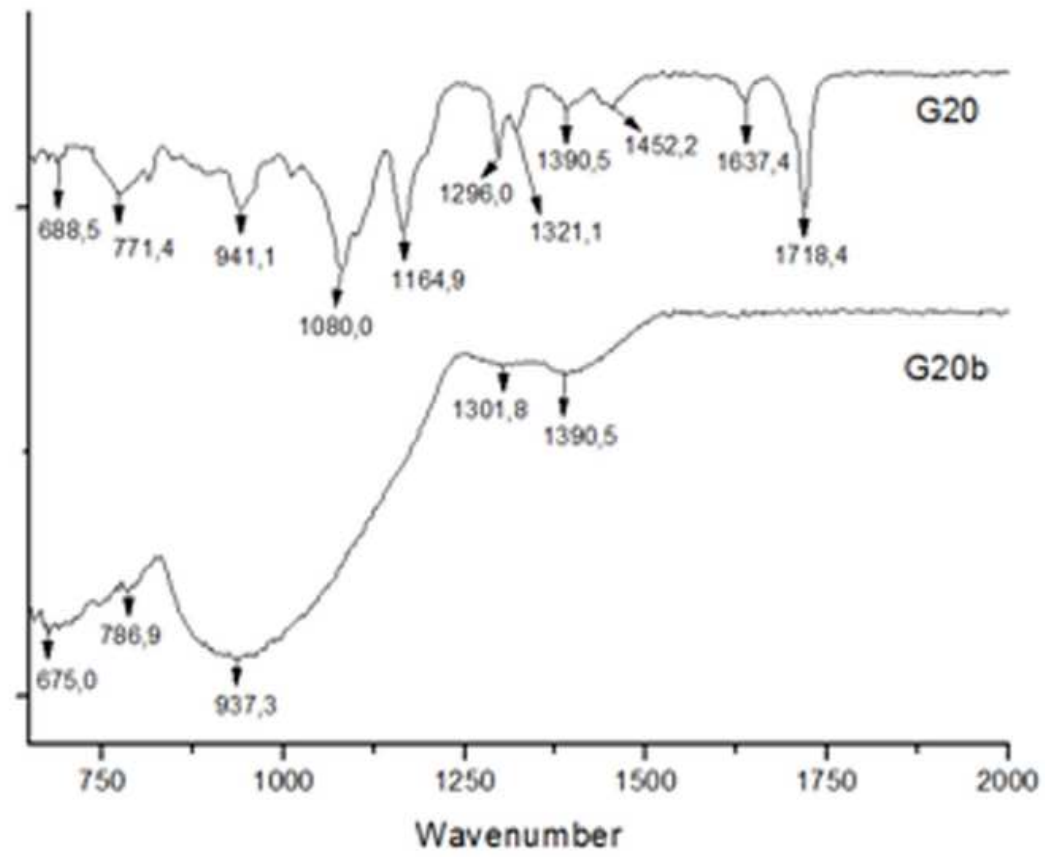

a)

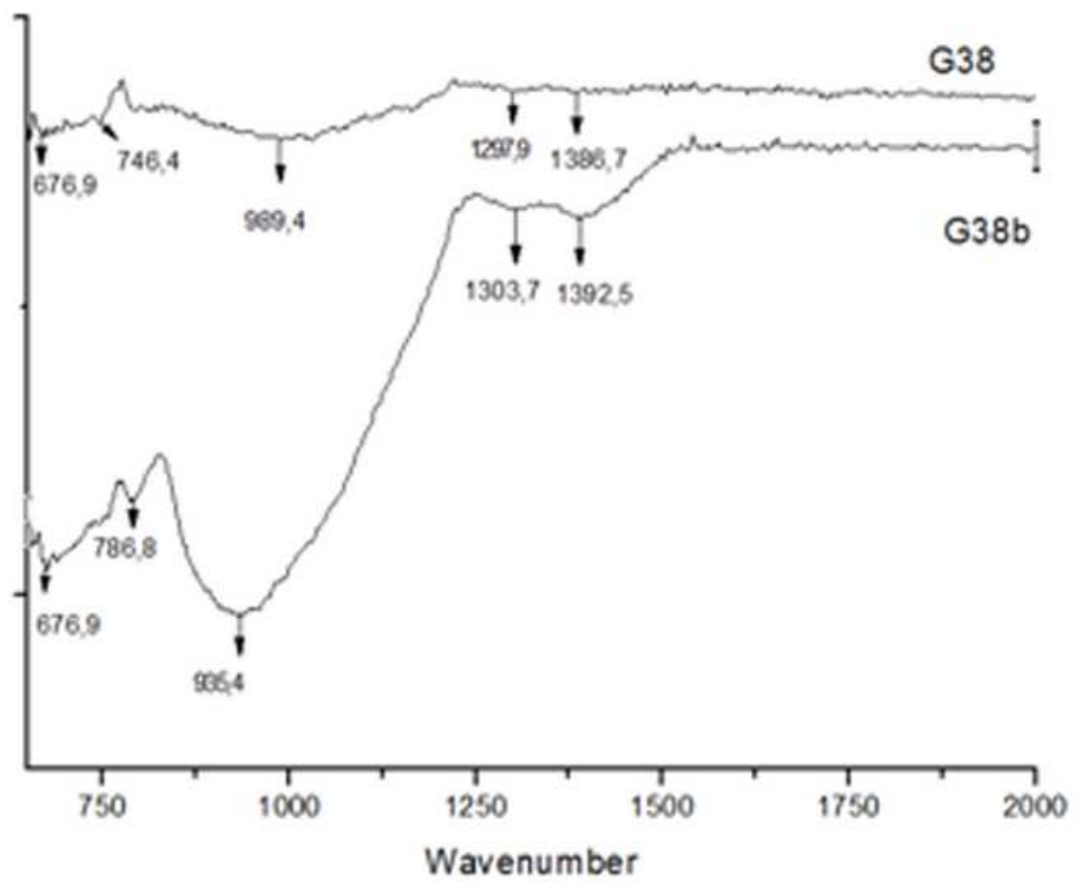

b) 


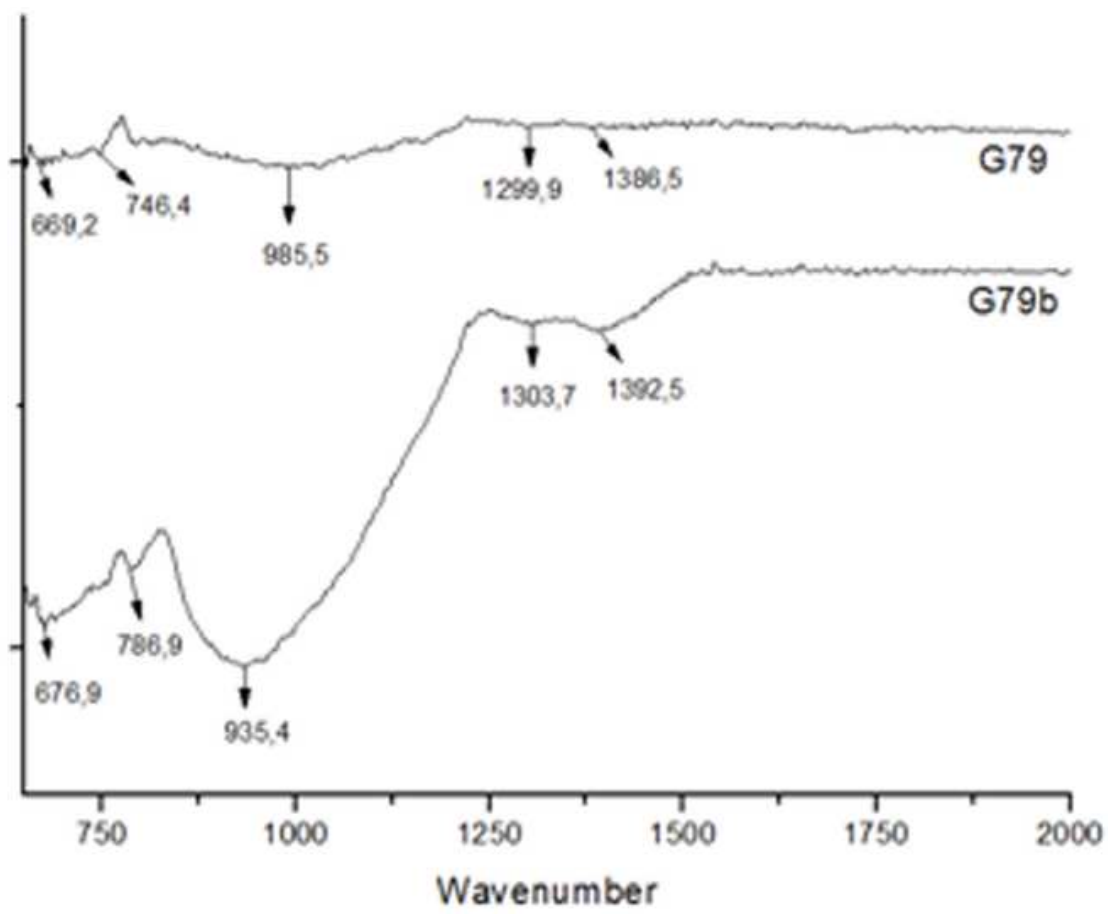

c)

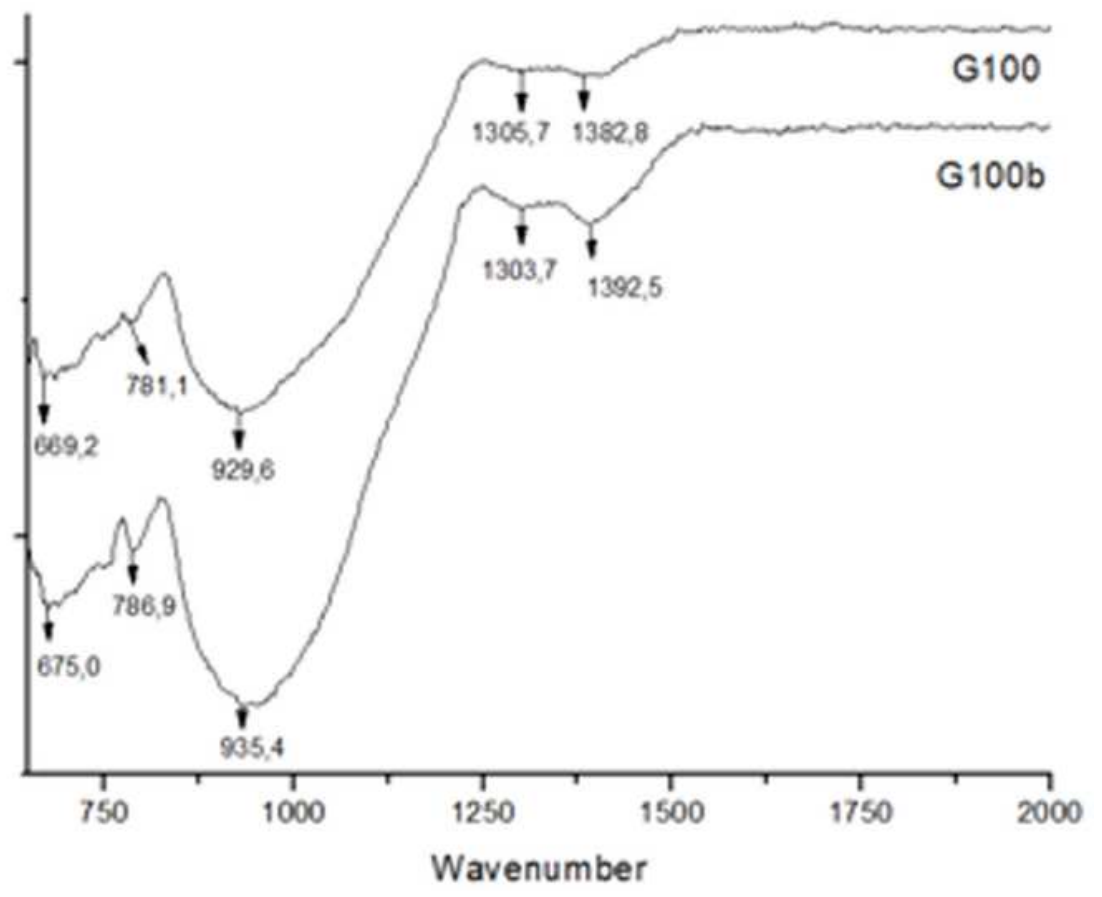

d) 